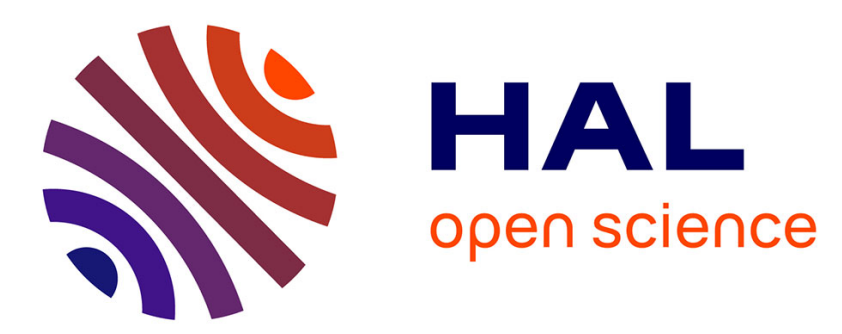

\title{
LTE-A Random Access Channel Capacity Evaluation for M2M Communications
}

\author{
Soukaina Cherkaoui, Inès Keskes, Hervé Rivano, Razvan Stanica
}

\section{To cite this version:}

Soukaina Cherkaoui, Inès Keskes, Hervé Rivano, Razvan Stanica. LTE-A Random Access Channel Capacity Evaluation for M2M Communications. WD 2016 - 8th IFIP Wireless Days, Mar 2016, Toulouse, France. 10.1109/WD.2016.7461480 . hal-01312768

\section{HAL Id: hal-01312768 \\ https://hal.inria.fr/hal-01312768}

Submitted on 9 May 2016

HAL is a multi-disciplinary open access archive for the deposit and dissemination of scientific research documents, whether they are published or not. The documents may come from teaching and research institutions in France or abroad, or from public or private research centers.
L'archive ouverte pluridisciplinaire HAL, est destinée au dépôt et à la diffusion de documents scientifiques de niveau recherche, publiés ou non, émanant des établissements d'enseignement et de recherche français ou étrangers, des laboratoires publics ou privés. 


\title{
LTE-A Random Access Channel Capacity Evaluation for M2M Communications
}

\author{
Soukaina Cherkaoui*, Inès Keskes ${ }^{\dagger *}$, Hervé Rivano*, Razvan Stanica* \\ ${ }^{*}$ Univ Lyon, INSA Lyon, Inria, CITI \\ F-69621, Villeurbanne, France \\ \{firstname.lastname\}@inria.fr \\ ${ }^{\dagger}$ Ecole Nationale d'Ingénieurs de Tunis, ENIT Tunis, Tunisia
}

\begin{abstract}
The densification of the urban population and the rise of smart cities applications foster the need for capillary networks collecting data from sensors monitoring the cities. Among the multiple networking technologies considered for this task, cellular networks, such as LTE-A, bring an ubiquitous coverage of most cities. It is therefore necessary to understand how to adapt LTE-A, and what should be the future $5 \mathrm{G}$ architecture, in order to provide efficient connectivity to Machine-to-Machine (M2M) devices alongside the main target of mobile networks, Human-toHuman devices. Indeed, cellular random access procedures are known to suffer from congestion in presence of a large number of devices, while smart cities scenarios expect huge density of M2M devices. Several solutions have been investigated for the enhancement of the current LTE-A access management strategy. In this paper, we contribute to the modeling and computation of the capacity of the LTE-A Random Access Channel (RACH) in terms of simultaneous successful access. In particular, we investigate the hypothesis of piggybacking the payload of Machine Type Communications from M2M devices within the RACH, and show that M2M densities considered realistic for smart cities applications are difficult to sustain by the current LTEA architecture.
\end{abstract}

Index Terms-M2M, LTE-A, RACH, MTC, Random Access, Capacity

\section{INTRODUCTION}

While many academic studies in the last decade have considered that wireless sensor networks are multi-hop, short range and low power, there is currently a strong push promoting cellular networks for collecting Machine-to-Machine (M2M) data [1]. Dedicated M2M cellular infrastructure are rising, e.g. $\operatorname{SigFox}^{1}$ and $\mathrm{LoRa}^{2}$, but address only very low throughput (few bytes per minutes) and mostly asymmetric applications. Traditional mobile operators are trying to take their share of the market, taking benefit from their ubiquitous coverage of cities and their ability to manage secured and authenticated communications. Besides, the background traffic of recent smartphones (e.g. application notifications, system synchronization) is very close to Machine Type Communication (MTC) traffic, and it is already causing resource management issues. As a consequence, the 2014 Ericsson mobility report [2] notes that more than 200 million cellular M2M devices were in used at the end of 2013. However, only $1 \%$ of

\footnotetext{
1 http://www.sigfox.com/en 2 http://lora-alliance.org
}

USA deployed M2M devices use the LTE network, while $64 \%$ of active M2M devices are still connected via GPRS-EDGE. As CDMA-based $3 \mathrm{G}$ is not suitable to low power operations [1], exploiting the existing LTE infrastructure and preparing the future $5 \mathrm{G}$ standards for M2M access is therefore a very important challenge.

All the cellular technologies share a common characteristic in the use of slotted Aloha [3] as the pillar element of the Random Access Channel (RACH). Even though slotted Aloha has been extensively studied in terms of achievable throughput [4], the literature is missing a proper modeling of the access capacity. In particular, the question we ask is how does an LTE-A eNodeB react when facing a large number of uplink requests?

Although the 3GPP LTE-A standard is incrementally including some support for MTC communications, the modern cellular system has been basically designed, engineered and managed to afford access to a reasonable number of Humanto-Human $(\mathrm{H} 2 \mathrm{H})$ communications, with high dowlink data rate. In contrast, $\mathrm{M} 2 \mathrm{M}$ applications require connections from a significant number of devices, mostly for uplink traffic, each communication having a low volume, and the interarrival times being conditioned by duty-cycle mechanisms [5]. The usual optimization of the infrastructure may therefore be irrelevant for supporting $\mathrm{M} 2 \mathrm{M}$ communications. In particular, the LTE-A Random Access (RA) procedure organizes the contention for access both physically, on time-frequency resources, and logically, on available signatures, which makes the collision detection challenging. When the number of user equipments (UE) is too large, the access may be denied to virtually all of them. This kind of congestion is classic in $\mathrm{H} 2 \mathrm{H}$ communication, when crowds gather at the same place for exceptional events. With the deployment of MTC devices, such density of UEs can become a regular situation.

Our main contribution in this study is providing a new modeling of the RA procedure that abstracts the physical layer and focuses on the impact of the traffic arrival rate and on the number of M2M devices. This model is used in extensive simulations, to derive engineering insights on the configuration of the RACH. The parameters showing the highest impact are the number of RA opportunities in which UEs can compete for access, and the number of available preambles, which gives 
the size of the contention space. The trade-offs between these parameters show that each configuration presents a threshold on the number of users after which the success ratio drops very quickly, requiring an adaptation of the RACH settings to the number of users.

The rest of the paper is structured as follows. In Section II, we give a brief overview of M2M communications and of the random access procedure in LTE-A. Section III introduces our RACH model, while Section IV is dedicated to the analysis of our simulation results and the deduced engineering insights. Section V positions our contribution among the literature of the domain, before concluding remarks in Section VI.

\section{RACH FOR M2M COMMUNICATION}

Communication in a mobile network follows two major procedures: a resource allocation demand is first transmitted by the UE which, if successful, is followed by the actual data transmission phase, using dedicated resources allocated by the eNodeB (eNB) to the UE. This procedure was designed specifically for $\mathrm{H} 2 \mathrm{H}$ traffic, which is bursty by nature, and it is based on the notions of session and call, activities that keep the user involved for a relatively long time and necessitate the exchange of a series of messages with the network.

$\mathrm{M} 2 \mathrm{M}$ traffic is essentially heterogeneous and applicationdependent, which makes it uneasy to classify into a specific category. However, it is common to smart metering and reporting applications to generate and transmit periodically very low amount of data [6]. This means that the M2M UEs have to go through a signaling-heavy RA procedure, described below, simply to transmit one message to the network.

Therefore, the idea of piggybacking the M2M data transmission within one of the RA procedure messages is tempting and it is now considered as the best solution for this type of traffic [7]. This means that the M2M data is transmitted on the shared resources of the $\mathrm{RACH}$, and raises questions regarding the capacity of the RACH, which was not designed for these purposes. In the following, we detail the RA procedure, used by UEs competing for channel access. This competition takes place on two dimensions: the RACH resource blocks (RB), and the preamble signatures.

An eNB contains a pool of predefined orthogonal signal patterns, denoted preamble signatures and divided into two groups: contention-based and contention-free signatures. The explanation for this division is that, for some operations where the user is already connected to the network, such as handover or new downlink data arrival, the UE is allocated directly a contention-free signature. However, in most cases, and especially for M2M traffic, the UE is idle before a transmission and therefore needs to go through the RA procedure, using contention-based preambles. The RA procedure consists of four successive steps:

- Step 1 (UE $\rightarrow$ eNB): Preamble transmission.

A user needing network access randomly selects and transmits a preamble among those available for the contention-based procedure. At this step, no additional information is sent by the UE. In particular, the eNB is not informed yet of the reason for the resource request. It is important to note that the preambles can be transmitted only during an RA slot, and the number of RA slots per LTE-A frame is decided by the eNB and broadcast to the UEs in the system information block. As discussed in Section IV, the UE can decide to increase the number of RA slots, but this will happen at the expense of data slots.

\section{- Step $2(e N B \rightarrow U E)$ : Random access response.}

If a preamble has been correctly decoded, the eNB acknowledges it by sending a random access response (RAR). Since the eNB has simply received a predefined signal pattern, it has no knowledge on the identity of the UE making the request. Therefore, the RAR is addressed to a Random Access Radio Network Temporary Identifier (RA-RNTI), which identifies the $\mathrm{RB}$ on which the preamble was decoded. As the transmitting UE implicitly knows the RB it used to transmit the preamble, it will also be able to interpret the acknowledgment. A back-off indicator can also be included in the RAR, to be used by the UEs in case of a failed transmission in Step 3. A device expects to receive the RAR within a configured time window, or else it deduces that its preamble was not decoded and goes back to Step 1, retransmitting with a higher transmitting power (i.e. the power ramping mechanism) during the next RA opportunity. It is noteworthy that the RAR does not directly help solving collisions: if several users have sent the same preamble in the same RA slot, and if the eNB has decoded it, they will all receive the same RAR and consider they have been granted uplink resources for message transmission in Step 3.

\section{- Step 3 (UE $\rightarrow$ eNB): Connection request message.}

This message contains the connection request cause, such as a Radio Resource Control (RRC) connection request, tracking area update or scheduling request. In this step, the UEs send for the first time their real identity to the eNB. For M2M traffic, this is the message considered for piggybacking the application data, as explained above. We note that users who collided on preamble selection in Step 1 are granted the same uplink resources in Step 3, therefore their transmissions will interfere. This interference may lead all the colliding UEs to restart the complete RA procedure. Nevertheless, if one user has a much better channel quality than the others, its message will be successfully decoded, a phenomenon denoted as the capture effect.

\section{- Step 4 (eNB $\rightarrow U E)$ : Contention resolution.}

This message conveys the identity of the decoded UE in the previous step. If an UE that transmitted a message in the previous phase can not decode the contention resolution message, or if it does not find its identity within this message, the UE stops the RA procedure and restarts another one from Step 1. On the contrary, an UE retrieving its identity within this message marks a successful access procedure and can use the dedicated uplink resources granted by the eNB in the message. In the case of M2M, this message is simply used for contention resolution, as an acknowledgement of the reception of the information piggybacked at Step 3 . 


\section{RA PROCEDURE MODEL}

In this section, we present our modeling of the RACH, our main assumptions and the default values of some parameters. We note that we evaluate the performance of the RA procedure from a network perspective. We therefore make an abstraction of lower protocol layers and the physical environment. In particular, we do not model precisely the position of the UEs, nor the radio propagation, and account for transmission failures and capture effect as input parameters of the model. Practically, the entire physical layer is represented by three parameters: $p_{d}, p_{c}, p_{r}$, discussed below. The proposed model is summarized in Fig. 1.

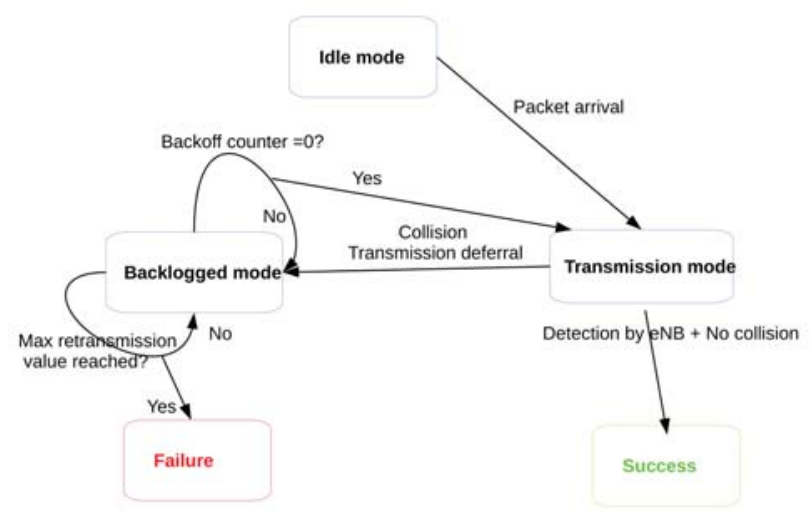

Fig. 1: LTE-A random access procedure model.

\section{A. Assumptions and model description}

One cell of 3GPP LTE-A is considered serving a set of M2M devices that are perfectly time-synchronized. UEs can be in either one of the three following states.

- Idle (I-mode): UEs with an empty transmission buffer.

- Transmitting mode (T-mode): users trying to send a message.

- Backlogged (B-mode): UEs waiting to retry after a transmission failure.

We assume that the traffic is periodic, each UE having one message arrival per period. The message is considered small enough to be piggybacked in the Step 3 of the RA procedure, without segmentation. If, due to buffering and transmission failures, a UE has $x$ messages in its buffer at a given time, it will perform $x$ times the RA procedure and send one message at a time. This is an obvious under estimation of the throughput in our model, but a better understanding of the access capacity of the cell.

Each period contains a number $O p p$ of RA opportunities. It is important to note that this gathers in one value two major parameters: the duration of the M2M application period, and the number of RA slots per LTE-A frame. On the one hand, the periodicity of the M2M traffic depends on the application: uploading vehicular data for fleet monitoring requires short periods, in the order of $100 \mathrm{~ms}$ [8], while smart metering applications produce one message every few minutes [6]. On the other hand, as mentioned in Sec. II, the number of RA slots per LTE-A frame is decided by the network, and can even change dynamically [9]. These two parameters are combined in one input value for our model.

The message arrivals at different UEs are randomly distributed over the application period. Upon message arrival, or when the transmission buffer is not empty, I-mode UEs switch to T-mode and start their RA procedure at the next RA opportunity. In each opportunity, T-mode UEs randomly select one of the available preambles and send it to the eNB. The size of the pool of available preambles for contention-based access is denoted $K$. The transmission of the preamble is detected by the eNB with probability $p_{d}$, which accounts for the possible transmission failures due to radio propagation issues. In our model, $p_{d}$ is independent and identically distributed for all the selected preambles, meaning that the preamble detection probability does not depend on the number of UEs having selected it.

UEs with undetected preambles move directly to B-mode. In the case of the detected preambles, the RA procedure continues and several possibilities arise:

i) The same preamble was selected by three or more UEs. In this case, we consider the RA procedure fails for all the nodes. This can happen either because the eNB realizes that multiple UEs transmitted the same preamble in Step 2, and therefore it does not replies with an RAR, or because the UEs reach Step 3 and transmit their messages at the same time, resulting in a collision. Practically, we consider that the interference is too important for the capture effect to allow the decoding of any message at the eNB.

ii) The same preamble was selected by two UEs. When this happens, the capture effect produces with probability $p_{c}$, meaning that the transmissions of the two UEs are received at the eNB with so different power levels that the eNB is able to detect and decode the message of the strongest signal. In our model, we have a probability $p_{c}$ that one of the UEs, randomly chosen between the two, succeeds and moves in I-mode, while the second UE restarts the RA procedure, by moving to B-mode. We note that the opposite event, arriving with probability $1-p_{c}$, is that both UEs fail and move in B-mode.

iii) The preamble was selected by only one UE. In this event, we only account for possible radio propagation problems, and the UE succeeds its transmission with probability $p_{r}$.

As explained, non-successful UEs are put in B-mode. They have the chance to retry their transmission after a backoff window, of maximum value $b_{\max }$. A maximum number of retrials is allowed and denoted $r_{\max }$. If $r_{\max }$ is reached, the access procedure fails. For the sake of simplicity and without losing precision on our estimation of the access capacity, we mingle the retransmissions in Step 1 with those in further steps, although the LTE-A standard distinguishes them for more detailed UE management.

\section{B. Parameter settings}

Our model relies on a set of parameters that are summarized in Tab. I. One can distinguish two kinds of parameters: those that describe our abstraction of the radio environment, and those describing the configuration of the system. 


\begin{tabular}{|l|l|l|}
\hline Notation & Description & $\begin{array}{l}\text { Default } \\
\text { value }\end{array}$ \\
\hline$p_{d}$ & $\begin{array}{l}\text { Detection probability of preamble } \\
\text { transmission at Step 1 }\end{array}$ & 0.95 \\
\hline$p_{r}$ & $\begin{array}{l}\text { Reception probability of transmis- } \\
\text { sions at Step 2 and 3 }\end{array}$ & 0.9 \\
\hline$p_{c}$ & $\begin{array}{l}\text { Capture probability at Step 3 if } \\
\text { collision on preamble }\end{array}$ & 0.8 \\
\hline \hline$b_{\max }$ & $\begin{array}{l}\text { Maximum value of backoff win- } \\
\text { dow }\end{array}$ & 5 \\
\hline$r_{\max }$ & \begin{tabular}{l} 
Number of allowed retransmissions \\
\hline$K$
\end{tabular} & Number of available preambles \\
\hline$O p p$ & Number of opportunities per period & 48 \\
\hline
\end{tabular}

TABLE I: Parameter settings and default values.

The first group consists of the three probabilities defined above $\left(p_{d}, p_{r}, p_{c}\right)$, that account for the effects of the physical layer, environment, and UE distribution. One could argue that these simple parameters are not enough to account for such a complex set of physical phenomenon. However, since we are not interested in the physical capacity of the RACH, but on the number of UEs that can be granted access in one period, we believe this aggregated and abstract modeling of the environment is enough. On reasonable ranges of values for these parameters, the impact of each probability is weak whatever the number of UEs. In the following, they are considered fixed with the assigned values in Tab. I.

The second group of parameters consists of the system settings that have to be set up by the operator. Most of them have default or advised values that are defined by $3 \mathrm{GPP}$, such as the backoff window or the maximum number of restransmissions. For the number of available preambles, 64 preamble signatures are defined by the standard. However, some of these preambles are reserved for contention-free access (see Sec. II), so not all of them are usable in the RA procedure. Finally, the LTE-A standard defines a set of valid frequencies of RA opportunities ranging between 0.05 to $1 \mathrm{RA}$ opportunities per millisecond Nevertheless, we remind that the time basis of our model is the periodicity of the application messages, not the one of the LTE-A frames. We are therefore interested in $O p p$, the number of opportunities per period, which does not only depend on the RA opportunities, but also on the application traffic model. We remark that, for the parameters in this second group, default values are provided in Tab. I, but the impact of each parameter is also analyzed.

\section{RESUlts AND DISCUSSION}

Assuming some essential in-home M2M applications, coupled with house density and cell size, an urban area cell should support in average 18000 devices [6]. Therefore, in order to investigate the capacity limits of the LTE-A RACH, our simulations are carried with more than 1000 devices. For the rest of the paper, unless specified, we consider the default values for parameters given in Tab. I.

\section{A. Impact of the number of available preambles}

We begin by studying the impact of the number of available preambles on the UE RACH success ratio, in Fig. 2. Our results show major difficulties in carrying the M2M traffic of more than 2000 UE with an acceptable success ratio, even

\begin{tabular}{|l|l|l|l|l|l|}
\hline $\begin{array}{l}\text { Number of } \\
\text { available } \\
\text { preambles }\end{array}$ & 100 & 200 & 300 & 400 & 500 \\
\hline Success ratio & 0.00059 & 0.065 & 0.41 & 0.87 & 0.97 \\
\hline
\end{tabular}

TABLE II: Success ratio for 10000 UEs with different numbers of available preambles.

when all the 64 available preambles are dedicated to M2M contention-based random access.

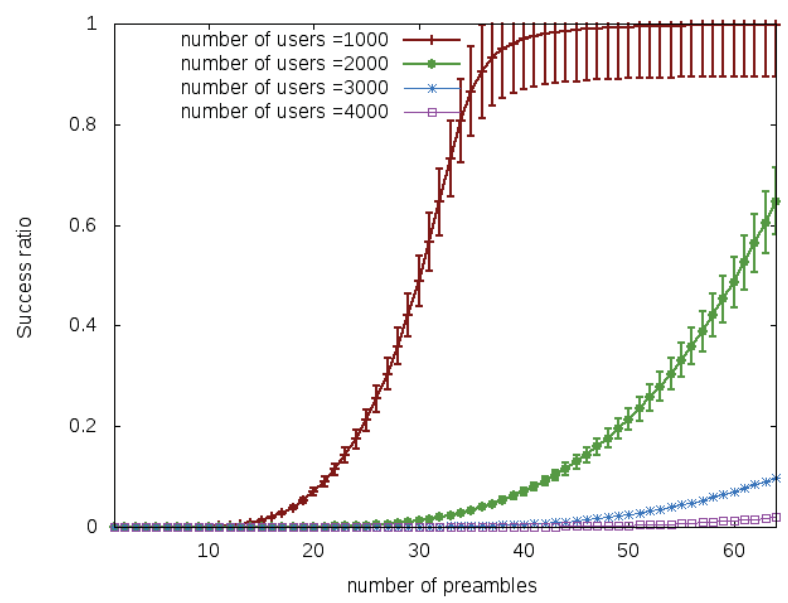

Fig. 2: Impact of the number of available preambles on the UE success ratio (95\% confidence intervals are shown).

Nevertheless, one must keep in mind that the number of preambles available for an eNB should be shared between contention-free and contention-based access. Also, the contention-based set could be separated according to the user type, between M2M and $\mathrm{H} 2 \mathrm{H}$ UEs. Considering that as many as 40 preambles are required in order to obtain an M2M success ratio higher than 0.95 for 1000 M2M UEs, even the support of this relatively small number of devices seems questionable in a real deployment.

As explained above, high densities of M2M devices are expected, especially in smart city scenarios. Therefore, in Tab. II we show that supporting 10000 M2M users per cell requires the eNB to provide only for these UEs almost 7 times more preambles than the value currently set by the standard, in order to guarantee an efficient success rate of 0.97 .

\section{B. Impact of the number of opportunities per period}

The opportunities represent the RBs where a user is allowed to transmit its access request. Therefore, the more opportunities a period is divided into, the wider the random access contention space is. Thus, increasing $O p p$ reduces the number of collisions and improves the success ratio, as shown in Fig. 3.

Increasing the number of opportunities per period can be achieved in two different ways. The frequency of RA opportunities can be increased, as explained in Sec. III-B, but this comes at the expense of less RBs for data in the LTE-A frame. This means that, adding more RACH slots to increase the success ratio of M2M UEs can have a significant negative effect on regular $\mathrm{H} 2 \mathrm{H}$ UEs, who mostly use dedicated RBs. The transmission period of the M2M application can also be increased, to allow more access opportunities to the 


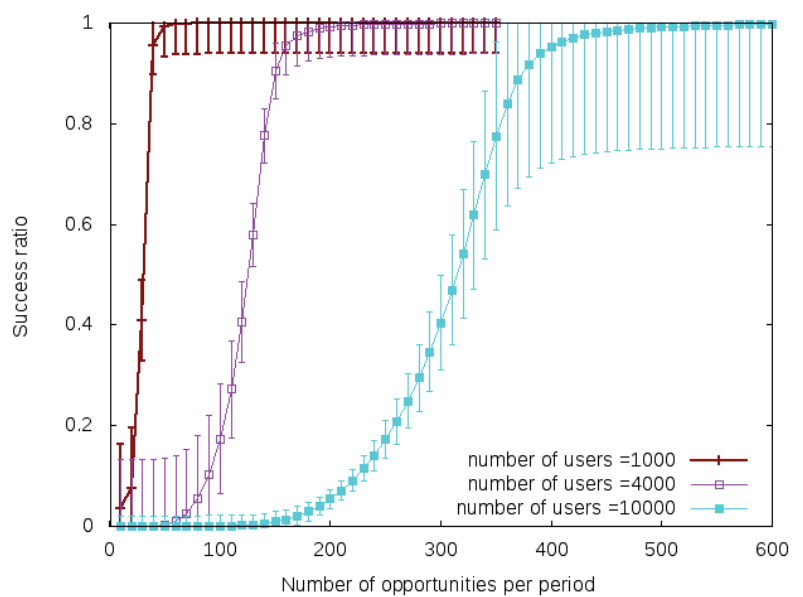

Fig. 3: Impact of the number of opportunities per period on the success ratio (95\% confidence intervals are shown).

users. However, the transmission period generally comes as a constraint, being dictated by application requirements.

The impact of the $O p p$ parameter is significant. For example, if we consider a normal scenario today, with one RA opportunity every LTE-A frame, the results in Fig. 3 can be understood as follows: if 48 preambles are dedicated to M2M traffic, the LTE-A RACH can support the access of up to 1000 UEs running an application with a transmission period of 500ms, and up to 10000 UEs if the application has a transmission period of $4.5 \mathrm{~s}$.

\section{Impact of the number of retransmissions}

If the RA procedure fails, the UE will enter a back-off phase before attempting the access again. The number of attempts that are allowed, $r_{\max }$, is decided by the network, and communicated to the UEs. The impact of $r_{\max }$ on the UE success ratio is shown in Fig. 4.

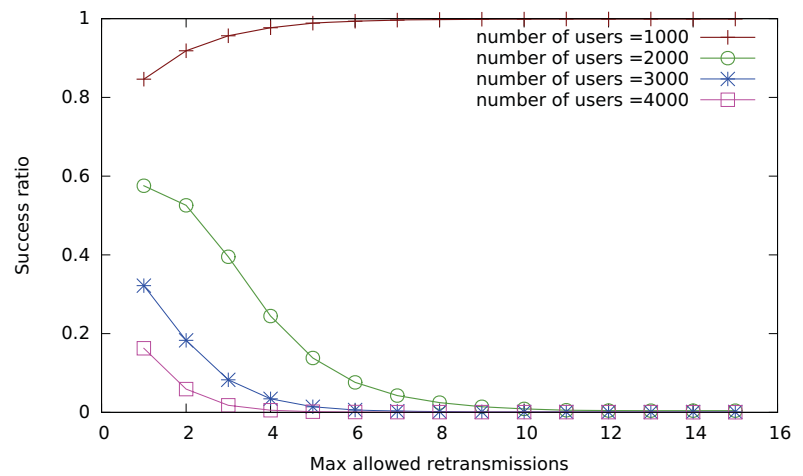

Fig. 4: Impact of the number of allowed retransmissions on the success ratio.

Two opposite behaviors can be noticed, depending on the number of UEs in contention for access. For less than 1000 M2M users, increasing $r_{\max }$ results in a higher success ratio. However, when more than 2000 UEs are present, authorizing more retransmissions simply leads to an even higher RACH congestion, drastically reducing the success ratio.
A high number of retransmissions can also result in larger transmission delays. As a consequence, a situation might occur where a new message arrives at the MAC layer for transmission before the UE can finalize the RA procedure for the previous message. In our model, we consider that these messages will use independent access procedures, whereas in a practical deployment they could be aggregated, or even transmitted on dedicated RBs, outside the RACH.

\section{Impact of the backoff window size}

To highlight the impact of the backoff window configuration, we choose a less congested situation than in the previous scenarios, by setting the number of preambles to 60 , while the default values are used for the other parameters.

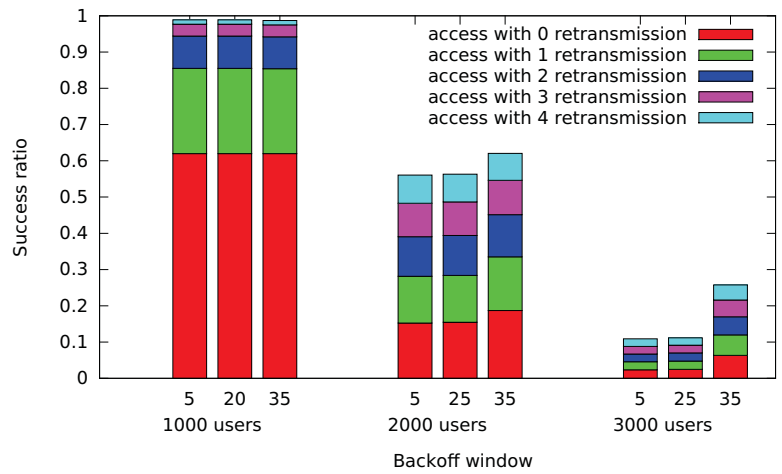

Fig. 5: Backoff impact on success ratio and distribution of the successful transmission attempt.

The success ratio for different backoff windows is shown in Fig. 5, where the successful transmission for the different UEs is also depicted. For example, we can notice that the backoff window does not impact significantly the success distribution for the $1000 \mathrm{M} 2 \mathrm{M}$ users case, where $60 \%$ of the accesses are done without retransmission, regardless the backoff window size. However, in heavily congested scenarios, e.g. 3000 M2M users, increasing the back-off window can bring important improvements, a back-off window of 35 RA slots almost doubling the success ratio compared with the default value.

\section{E. Preamble dimensioning use case}

From the results above, two parameters are noticed to have a major impact on the performance of the RA procedure: the number of available preambles, and the number of opportunities per period. As explained, this second parameter is not entirely up to the operator, as it also depends on the application requirements. We therefore focus on the number of preambles, by analyzing the minimum number of signatures required for $\mathrm{M} 2 \mathrm{M}$ users in order to guarantee a targeted success ratio. These results are presented in Fig. 6, where the traffic arrival is represented in terms of number of UEs attempting the access in a given RA opportunity.

The results outline a trade-off between the acceptable performance and the available preamble resources. For example, improving the success ratio from 0.8 to 0.999 costs the addition of at least 20 more preambles for a traffic intensity 


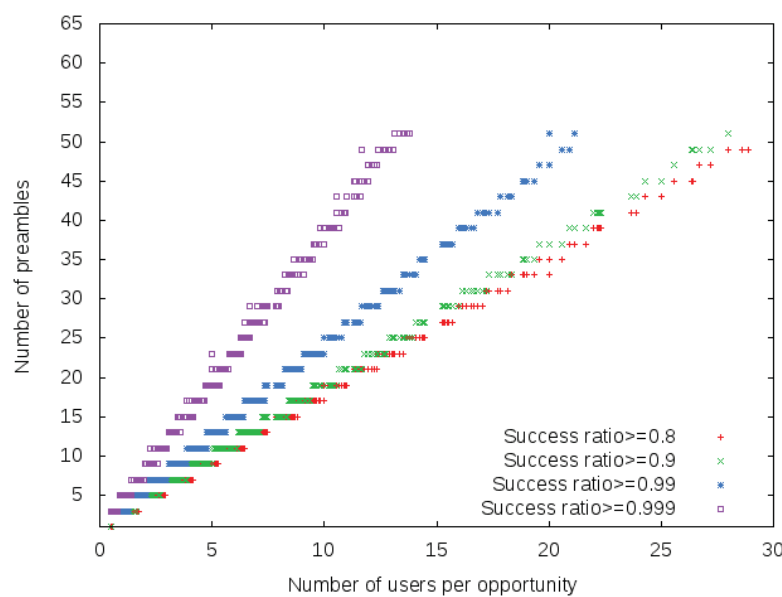

Fig. 6: Minimum number of preambles required for a predefined success ratio, as a function of the number of users per opportunity.

of 10 users per opportunity, while only 5 additional preambles are required to reach a 0.99 success rate.

Overall, we observe that, using the current values of the standard, the capacity of the RACH is one order of magnitude smaller than the number of M2M devices that are expected in a smart city scenario. This problem can be alleviated by increasing the number of available preambles or RA opportunities for M2M traffic, but such solutions should be carefully designed, in order to minimize the impact on the regular $\mathrm{H} 2 \mathrm{H}$ traffic.

\section{RELATED WORKS}

As the $\mathrm{H} 2 \mathrm{H}$ traffic generally uses dedicated resources, most of the studies in the literature focus on the performance of the cellular downlink. The cellular uplink in general, and the RACH in particular, have been relegated to a second level. For example, state-of-the-art LTE simulation frameworks, such as LTE-Sim [10] do not implement the random access procedure. With the rise of M2M traffic, which is mostly uplink, a few studies tackled the subject of the LTE capacity at a physical layer, e.g. [11]. However, physical layer metrics are difficult to translate in an access capacity and require as input a very detailed knowledge of the environment and of the UE placement.

The first attempts to evaluate the LTE RACH capacity in terms of successful UE access ([7], [12]) were presented in the 3GPP working groups. However, these studies consider a constant collision probability, independent of the number of UEs or of the available resources. Analytical models of the RA procedure were also proposed (e.g. [13], [14]), where the UE outage probability is computed. These studies also make assumptions that are difficult to verify on the collision probability, such as an exponential dependence on the number of preambles in [13]. Also, phenomena difficult to represent analytically, such as the capture effect, are ignored. Finally, several studies start from the observation that RACH congestion is produced by M2M devices, and propose different mechanisms to alleviate this problem [15], without actually quantifying it.

\section{CONCLUSIONS}

Support of short information messages generated by M2M applications is identified as one of the key challenges for $4 \mathrm{G}$ and beyond wireless networks. In this paper, we propose a model for the RA procedure in LTE-A networks. By abstracting the physical layer parameters, we are able to model in detail the RA procedure, without any supplementary assumptions on the collision probability, while at the same time taking into account complex phenomena such as the capture effect. This allows us to provide an estimation of the access capacity on the LTE-A uplink, and highlight the difference between the number of M2M UEs that can be supported by the RACH and the number of devices foreseen by $\mathrm{M} 2 \mathrm{M}$ applications.

\section{ACKNOWLEDGMENT}

This work has been carried out in the framework of IDEFIX project, funded by the ANR under the contract number ANR13-INFR-0006, the ADR Green of the Alcatel-Lucent/INRIA common laboratory, and the Inria Project Lab CityLab@Inria.

\section{REFERENCES}

[1] A. Bartoli, M. Dohler, J. Hernandez-Serrano, A.A. Kountouris, and D. Barthel, "Low-Power Low-Rate Goes Long-Range: The Case for Secure and Cooperative Machine-to-Machine Communications," Proc. IFIP WCNS, Valencia, Spain, May 2011.

[2] Ericsson, "Ericsson Mobility Report" Technical Report, June 2014. [Online]. Available: http://www.ericsson.com/res/docs/2014/ericssonmobility-report-june-2014.pdf

[3] J. Metzner, "On Improving Utilization in ALOHA Networks," IEEE Transactions on Communications, vol. 24, no. 4, pp. 447-448, Apr. 1976.

[4] C. Namislo, "Analysis of Mobile Radio Slotted ALOHA Networks," IEEE Transactions on Vehicular Technology, vol. 33, no. 3, pp. 199-204, Aug. 1984.

[5] M.Z. Shafiq, L. Ji, A.X. Liu, J. Pang, and J. Wang, "Large-Scale Measurement and Characterization of Cellular Machine-to-Machine Traffic" IEEE/ACM Transactions on Networking, vol. 21, no. 6, pp. 1960-1973, Dec. 2013.

[6] Vodafone, "RACH Intensity of Time Controlled Devices," 3GPP TSGRAN WG2, R2-102296, Beijing, China, Apr. 2010.

[7] 3GPP TR 37.868, "Study on RAN Improvements for Machine-type Communications," v11.0.0, Sep. 2011.

[8] S. Ancona, R. Stanica, and M. Fiore, "Performance Boundaries of Massive Floating Car Data Offloading," Proc. IEEE WONS, Obergurgl Austria, Apr. 2014.

[9] D. Kim, W. Kim, and S. An, "Adaptive Random Access Preamble Split in LTE," Proc. IWCMC, Cagliari, Italy, Jul. 2013.

[10] G. Piro, L.A. Grieco, G. Boggia, F. Capozzi, and P. Camarda, "Simulating LTE Cellular Systems: An Open-Source Framework" IEEE Transactions on Vehicular Technology, vol. 60, no. 2, pp. 498-513, Feb. 2011.

[11] T.D. Novlan, H.S. Dhillon, and J.G. Andrews, "Analytical Modeling of Uplink Cellular Networks" IEEE Transactions on Wireless Communications, vol. 12, no. 6, pp. 2669-2679, Jun. 2013.

[12] Ericsson, "LTE Random-Access Capacity and Collision Probability," 3GPP TSG-RAN WG1, R1-061369, Shanghai, China, May 2006.

[13] R.-G. Cheng, C.-H. Wei, S.-L. Tsao, and F.-C. Ren, "RACH Collision Probability for Machine-Type Communications", Proc. IEEE VTC Spring, Yokohama, Japan, May 2012.

[14] J.J. Nielsen, D.-M. Kim, G. Corrales Madueno, N.K. Pratas, and P. Popovski, "A Tractable Model of the LTE Access Reservation Procedure for Machine-Type Communications," arXiv:1505.01713, Aug. 2015.

[15] A. Laya, L. Alonso, and J. Alonso-Zarate, "Is the Random Access Channel of LTE and LTE-A Suitable for M2M Communications? A Survey of Alternatives," IEEE Communications Surveys \& Tutorials, vol. 16, no. 1, pp. 4-16, Jan. 2014. 